\title{
Ambiente físico y respuestas fisiológicas de ovinos bajo sombra en horas de máxima radiación
}

\author{
Reyes, J. ${ }^{\text {; }}$ Herrera, M. ' ; Marquina, J.R. ${ }^{2}$; Enjoy, D.D. ${ }^{1}$ y Pinto-Santini, L. ${ }^{1}$
}

'Instituto de Producción Animal. Facultad de Agronomía. Universidad Central de Venezuela. Maracay. Venezuela.

${ }^{2}$ Instituto Nacional de Investigaciones Agrícolas. Maracay, Venezuela.

\section{PALABRAS CLAVE ADICIONALES}

Bienestar animal.

Estrés calórico.

Temperatura superficial.

Sombra artificial.

West African.

\section{RESUMEN}

Con el objeto de describir la relación entre las variables climáticas y las respuestas fisiológicas, 20 ovejas fueron asignadas a: Con Sombra (CS): sombra artificial (12:00 - 14:00 h) en corral con techo de malla sombra (70\%); Sin Sombra (SS): sin ofrecimiento de sombra. Se determinó radiación solar (RS; W/m2), temperatura ambiental $\left({ }^{\circ} \mathrm{C}\right)$, humedad relativa $(\%)$, temperatura de globo negro (TGN; ${ }^{\circ} \mathrm{C}$ ) e índice de temperatura-humedad (ITH; unidades), temperatura superficial (TS; ${ }^{\circ} \mathrm{C}$; termómetro infrarrojo), temperatura corporal (TC; ${ }^{\circ} \mathrm{C}$; termómetro digital), frecuencia respiratoria (FR; mov/min; observación directa) y coeficiente de tolerancia al calor (CTC; unidades) (PROC MIXED; SAS).Adicionalmente, los datos fueron analizados por componentes principales (InfoStat/E, 2008).CS presenta una menor TS $(35,9)$ en comparación con SS $(38,9 ; \mathrm{P} \leq 0,01)$; igual ocurre en TC $(P \leq 0,01)$. La FR en CS $(112,3)$ fue superior a SS $(139,6 ; P \leq 0,01)$. El CTC fue mayor en SS $(7,8)$ vs. CS $(5,8 ; \mathrm{P} \leq 0,01)$, indicando mejor bienestar térmico en CS. La TS en lomo, cadera y/o costado presenta alta relación con TC. TGN $>42,8$ y de $R S>529,1$ podrían indicar reducciones del bienestar térmico. Basado en la TC y FR, el punto de quiebre del ITH en ovinos es $>80$.

\section{Physical environment and physiological responses in sheep under shade during hours of higher radiation}

\section{SUMMARY}

\section{ADDITIONAL KEYWORDS}

Animal welfare.

Heat stress.

Superficial temperature.

Artificial shade.

West African.

\section{INFORMATION}

Cronología del artículo.

Recibido/Received: 05.10 .2016

Aceptado/Accepted: 05.05.2018

On-line: 15.07.2018

Correspondencia a los autores/Contact e-mail:

liviapintosantini@gmail.com

\section{INTRODUCCIÓN}

En Venezuela, los sistemas de producción con ovinos son manejados en forma extensiva y/o semi-intensiva y presentan bajos niveles productivos. Tradicionalmente se ubican en zonas áridas y semi-áridas y en algunos estados llaneros; lugares estos con elevada temperatura ambiente (TA) y alta radiación solar glo- bal (RS), por lo que incluso ovejas de razas consideradas adaptadas al trópico como la oveja West African (Núñez-Domínguez et al. 2016, p. 256), presentan riesgo de sufrir de estrés calórico (EC).

En la actualidad no existe un índice de bienestar térmico en ovinos que permita su uso a nivel de campo. 
En términos climáticos, ha sido utilizado el índice de temperatura-humedad (ITH) (Thom 1959); sin embargo, se desconoce el punto de quiebre que represente riesgos de EC en ovinos tropicales (López et al. 2015, p. 143).

La evaluación fisiológica de los animales indica su nivel de tolerancia en situaciones de EC (López et al. 2015, p. 140; Cerqueira et al. 2016, p. 257). El coeficiente de tolerancia al calor (CTC) (Benezra 1954, p. 1015) que relaciona la temperatura corporal y la frecuencia respiratoria, ha sido utilizado en cabras (Rocha et al. 2009, p. 1166) y ovinos (Batista et al. 2014, p. 103; López et al. 2015, p. 141). Debido a la combinación de carga radiante, TA y velocidad del viento, la temperatura de globo negro (TGN) podría ser utilizada para medir el efecto del ambiente sobre parámetros fisiológicos en pequeños rumiantes bajo condiciones tropicales.

Aunque las investigaciones sobre efectos del EC en especies de interés zootécnico son abundantes, la cantidad de reportes de zonas templadas supera la cantidad de estudios desarrollados en el trópico y con animales tropicales. Tal diferencia es mayor si se compara la cantidad de estudios evaluando estrategias para mitigar los efectos del EC. Con la finalidad de hacer más aplicable la evaluación de situaciones de EC, este trabajo busca describir las relaciones entre elementos climáticos y respuestas fisiológicas de ovejas West African resguardadas en sombra artificial durante las horas de mayor RS.

\section{MATERIALES Y MÉTODOS}

El experimento se realizó en el Laboratorio Sección de Ovinos del Instituto de Producción Animal de la Facultad de Agronomía de la Universidad Central de Venezuela $\left(10^{\circ} 14^{\prime} 49^{\prime \prime} \mathrm{N}, 67^{\circ} 35^{\prime} 45^{\prime \prime} \mathrm{W}\right)$, localizado a $443 \mathrm{msnm}$, con clima seco tropical y precipitación de 600-800 mm anuales. La TA mínima y máxima es de 21,1 y $32,0^{\circ} \mathrm{C}$. La humedad relativa (HR) oscila entre 60 y 80\% (USICLIMA 2012). El experimento se realizó durante la transición entre la época seca y la época de lluvia (Abril-Mayo de 2015) y tuvo una duración de $32 \mathrm{~d}$.

Se emplearon 20 ovejas adultas vacías de la raza West African, manejadas en semi-estabulación, con pastoreo (Cynodon nlemfluensis) en las horas diurnas y confinamiento el resto del tiempo. Durante el estudio las ovejas pastorearon libremente entre las 08:00 y 12:00 $\mathrm{h}$ y luego eran trasladadas a uno de dos corrales, según el tratamiento: a) Tratamiento con sombra (CS) $(n=10)$ : corral de $30 \mathrm{~m}^{2}$ con sombra artificial (techo de malla sombra verde, $70 \%$ ); b) Tratamiento son sombra (SS) $(\mathrm{n}=10)$ : corral de $30 \mathrm{~m}^{2}$ sin ofrecimiento de sombra. Los corrales fueron construidos en un extremo de los potreros y las ovejas permanecieron en ellos por un periodo de $2 \mathrm{~h}$ (12:00 a 14:00 h), con acceso a pacas de heno y agua a voluntad.

Se caracterizó el microclima en CS y SS a través del registro de la TA $\left({ }^{\circ} \mathrm{C}\right), \mathrm{HR}(\%)$ y $\mathrm{RS}\left(\mathrm{W} / \mathrm{m}^{2}\right)$ medidas las $24 \mathrm{~h}$ del día, obtenidas a través de sensores electrónicos conectados a estaciones meteorológicas automáticas ubicadas en CS y SS. Adicionalmente, se midió la TGN mediante el empleo de un termómetro de bulbo negro construido según la metodología de Souza et al. (2002, p. 160) durante las horas de mayor RS. Como indicador de bienestar térmico basado en los elementos climáticos, se calculó el ITH. Los valores climáticos presentados durante la fase experimental se resumen en la Tabla I.

Culminado el periodo de permanencia bajo sombra o sol, según el tratamiento, se efectuaron las determinaciones de las variables fisiológicas. Se determinó la frecuencia respiratoria (FR; mov/min) cuantificando el número de movimientos del flanco del animal durante 15 segundos, para luego multiplicar el valor por cuatro. Posteriormente, cada animal fue cuidadosamente sujetado para la determinación de la temperatura superficial (TS) y la temperatura corporal (TC). La TS $\left({ }^{\circ} \mathrm{C}\right)$ se determinó sobre el pelaje de los animales, a través de un termómetro infrarrojo (Mini IR Thermometer; Extech Instruments), en nueve puntos: 1) lomo (región lumbar), 2) cadera (región de la grupa), 3) costado (triángulo del ijar), 4) vena nasal dorsal, 5) vena angular ocular, 6) frente (lóbulo frontal), 7) base del cráneo (región occipital), 8) extremidad anterior (radio/cubito), 9) extremidad posterior (tibia/peroné). La TC $\left({ }^{\circ} \mathrm{C}\right)$ se determinó con el uso de un termómetro digital Testo 110 y una sonda de inmersión/penetración, introducida durante 20 segundos a nivel del recto a una profundidad de $5 \mathrm{~cm}$.

Como índice de bienestar térmico basado en las respuestas fisiológicas se calculó el CTC utilizando la modificación propuesta por López et al. (2015, p. 142) para ovinos, utilizando la siguiente formula: CTC $=(\mathrm{TC} / 39)$ $+(\mathrm{FR} / 23)$. Este coeficiente señala que cuando el CTC es 2 , los índices fisiológicos de los animales estarían indicando que no hay EC o que el animal es tolerante al calor (Benezra 1954, p. 1015). Finalmente, a las 16:00 $\mathrm{h}$ las ovejas eran trasladas hasta su lugar de pernocta donde, siguiendo el manejo establecido por la unidad de producción, disponían de pacas de heno, alimento balanceado comercial, acceso a bloque multinutricional y agua a voluntad.

Los datos fueron analizados por ANAVAR para medidas longitudinales usando el procedimiento MIXED de SAS. Mediante la técnica de Littell et al. (1998, p. 1221), se seleccionó una estructura apropiada de varianza-covarianza. La comparación de medias fue efectuada por la instrucción PDIFF (SAS, 2012). El modelo incluyó el efecto de tratamiento (CS vs. SS), día de experimentación $(1,3,6 . .32)$ y su respectiva interacción. Adicionalmente, mediante análisis de componentes principales $(\mathrm{ACP})$, se describió la relación entre: a) las variables climáticas y la TC; b) TS y TC y c) TC, FR y las variables climáticas. Se consideró en todos los casos que el determinante de la matriz de correlación fuera $\sim 0$, y la correlación cofenética $\sim 1$, requisitos necesarios para garantizar la pertinencia del análisis multivariado y el adecuado uso del ACP, respectivamente (InfoStat/E, versión 2008; Di Rienzo et al. 2008, p. 182).

\section{RESULTADOS}

En la Tabla II se observa el efecto del uso de sombra sobre TS, TC, FR y CTC. La relación entre TC y las variables climáticas se observa gráficamente en la Figura 
1A. Debido a que la TS se determinó en diferentes puntos anatómicos, es de interés conocer cuál de éstos explica la mayor variabilidad en la TC (Figura 1B). La FR constituye una variable de fácil medición que no requiere la sujeción de los animales ni la adquisición de instrumentos de medición. En la Figura 1C se muestra la relación de la FR con las variables climáticas y TC. Los vectores medios de las variables que mejor explican las relaciones obtenidas con el uso del ACP se presentan en la Tabla III.

\section{DISCUSIONES}

El uso de sombra constituye una estrategia para reducir el efecto de la RS directa sobre rumiantes a pastoreo. Los resultados indican que el uso de sombra redujo la RS diaria en $147,2 \mathrm{~W} / \mathrm{m}^{2}(\mathrm{P} \leq 0,05)$ en comparación con el corral donde no se ofreció sombra, lo que representa un $71 \%$ de disminución (Tabla I). De igual forma, en las horas de mayor RS, la incidencia directa de la RS sobre las ovejas fue menor en un $85 \%$ en las áreas sombreadas. En concordancia, López et al. (2015, p. 142), utilizando toldos de lona como estrategia de sombreamiento, observaron una reducción de $91 \%$ en la RS. Los valores de ITH (Tabla I) superan en forma importante los umbrales de alerta por estrés en la mayoría de las especies animales ( $\geq 72$ unidades). Sin embargo, para ovinos de pelo este umbral de ITH podría no ser el punto de quiebre entre bienestar y EC. Trabajos recientes en caprinos establecen que valores $>84$ unidades de ITH implica que los animales están bajo condiciones de EC severo (Singh et al. 2016, p. 114)

A pesar de que la TC media se encuentra dentro del rango de la especie (De Sousa et al. 2008, p. 133), la FR es bastante elevada, lejos de los valores considerados normales para la especie por Batista et al. (2014, p. 105) y López et al. (2015, p. 141) (Tabla II). El CTC obser- vado en las ovejas supera el valor de referencia; sin embargo, las ovejas CS presentaron 2,5 veces el valor del índice, siendo menos estresadas que las ovejas SS.

Las variables climáticas con más relación con la TC son TGN, RS e ITH (CP1: 76\% de la variabilidad). La alta correlación entre TGN, RS e ITH podría indicar que la TGN, medida con termómetros artesanales como los elaborados en la presente experiencia, podría sustituir el uso de radiómetros y el cálculo del ITH a nivel de finca, constituyendo una valiosa herramienta práctica para la toma de decisiones operativas. Los umbrales obtenidos a partir de los vectores medios (Tabla III) indican que cuando la TGN y RS superan los 42,8 ${ }^{\circ} \mathrm{C}$ y $547,6 \mathrm{~W} / \mathrm{m}^{2}$, los animales pierden la capacidad de mantener la TC y alcanzan valores $\geq 39,5^{\circ} \mathrm{C}$. Debido a que los valores de ITH muestran diferenciales con la TC muy estrechos, se puede sugerir que el punto de quiebre de ITH en ovinos de pelo es $\sim 80$ unidades.

En la medida que la TA aumenta, la eficiencia en la pérdida de calor sensible disminuye, en función del menor diferencial entre la TS y la TA, por lo que valores de $\mathrm{TC} \geq 39,5^{\circ} \mathrm{C}$ podrían indicar ganancia de calor o menor eficiencia en los mecanismos de disipación de calor. Por sobre los $35^{\circ} \mathrm{C}$ de TA, las pérdidas de calor por las vías respiratorias en ovinos pueden llegar hasta un 60\% (Quesada et al. 2001, p. 1025). Sin embargo, el incremento de la actividad muscular debido al proceso respiratorio puede ser desventajoso debido a la considerable cantidad de calor generado por la musculatura respiratoria.

Batista et al. (2014, p. 106) observaron valores de TS cercanos a los $4^{\circ} \mathrm{C}$ por encima en ovejas expuestas al sol $\left(41,6^{\circ} \mathrm{C}\right)$ al compararla con ovejas bajo sombra $\left(37,7^{\circ} \mathrm{C}\right)$. En respuesta al EC se genera un incremento en el flujo sanguíneo hacia la superficie del animal, resultando un aumento en la TS, como mecanismo de disipación de calor a través de la piel. A pesar de que la capa de pelo representa la primera defensa contra la

Tabla I. Promedios ( \pm error estándar) de los elementos climáticos, índice de temperatura-humedad (ITH) y temperatura de globo negro durante las horas de mayor radiación solar (12:00-14:00h) en corrales con sombra (CS) y sin sombra (SS) (Means ( \pm standard error) of the climatic elements, temperature-humidity index(ITH) and black globe temperature during the hours with higher solar radiation (12:00-14:00h) in pens with shade (CS) and without shade (SS)).

\begin{tabular}{|c|c|c|c|c|}
\hline \multirow[t]{2}{*}{ Variable } & \multicolumn{2}{|c|}{ Promedio de variaciones diarias } & \multicolumn{2}{|c|}{ Promedios en horas de mayor radiación solar (12:00-14:00 h) } \\
\hline & CS & SS & CS & SS \\
\hline Humedad relativa (\%) & $65,6 \pm 0,8 a$ & $66,5 \pm 0,8$ a & $39,7 \pm 1,9 b$ & $43,4 \pm 2,1 \mathrm{a}$ \\
\hline Temperatura ambiente $\left({ }^{\circ} \mathrm{C}\right)$ & $26,1 \pm 0,1 \mathrm{a}$ & $26,3 \pm 0,1 \mathrm{a}$ & $32,9 \pm 0,3 a$ & $33,0 \pm 0,3 a$ \\
\hline Radiación solar global $\left(\mathrm{W} / \mathrm{m}^{2}\right)$ & $58,2 \pm 3,3 b$ & $205,4 \pm 10,3 a$ & $101,7 \pm 4,9 \mathrm{~b}$ & $659,9 \pm 42,0 \mathrm{a}$ \\
\hline \multirow[t]{2}{*}{ ITH (unidades) } & $74,0 \pm 0,1 \mathrm{a}$ & $74,3 \pm 0,2 a$ & $80,1 \pm 0,2 b$ & $80,7 \pm 0,1 \mathrm{a}$ \\
\hline & \multicolumn{4}{|c|}{ Temperatura de globo negro $\left({ }^{\circ} \mathrm{C}\right)^{*}$} \\
\hline Hora de medición & \multicolumn{2}{|c|}{ CS } & SS & Diferencia \\
\hline $12: 00 \mathrm{~h}$ & \multicolumn{2}{|c|}{$36,8 \pm 0,6 b$} & $45,1 \pm 0,7 a$ & $8,3 \pm 0,7$ \\
\hline $13: 00 \mathrm{~h}$ & \multicolumn{2}{|c|}{$37,5 \pm 0,4 b$} & $45,7 \pm 0,6 a$ & $8,2 \pm 0,5$ \\
\hline $14: 00 \mathrm{~h}$ & \multicolumn{2}{|c|}{$36,9 \pm 0,4 b$} & $44,9 \pm 0,6 a$ & $8,0 \pm 0,5$ \\
\hline Promedio & \multicolumn{2}{|c|}{$37,1 \pm 0,3$} & $45,3 \pm 0,5 a$ & $8,1 \pm 0,4$ \\
\hline
\end{tabular}

${ }^{a b}$ Diferente entre columnas indican diferencia significativa $(P \leq 0,05) ;{ }^{*}$ ab Diferente entre columnas indican diferencia significativa $(P \leq 0,01)$ 
Tabla II. Medias (土error estándar) de variables fisiológicas, coeficiente de tolerancia al calor y diferenciales de temperatura: temperatura superficial - temperatura ambiente (TS-TA), temperatura corporal - temperatura superficial (TC-TS) y temperatura ambiente - temperatura corporal (TA-TC) en corrales con sombra (CS) y sin sombra (SS) (Means ( \pm standard error) of physiological variables, coefficient of heat tolerance and temperature differentials: superficial temperature - ambient temperature (TS-TA), body temperature - superficial temperature (TC-TS) and ambient temperature - body temperature (TA-TC) in pens with shade (CS) and without shade (SS)).

\begin{tabular}{lccc}
\hline \multicolumn{2}{c}{ Tratamiento } & \\
\hline Variables & CS & SS & Diferencia SS-CS \\
\hline Temperatura superficial $\left({ }^{\circ} \mathrm{C}\right)$ & $35,9 \pm 0,1 \mathrm{~B}$ & $38,9 \pm 0,1 \mathrm{~A}$ & $+3,0 \pm 0,1$ \\
Temperatura Corporal $\left({ }^{\circ} \mathrm{C}\right)$ & $39,3 \pm 0,02 \mathrm{~B}$ & $39,4 \pm 0,03 \mathrm{~A}$ & $+0,1 \pm 0,02$ \\
Frecuencia Respiratoria (Mov/min) & $112,3 \pm 3,0 \mathrm{~B}$ & $139,6 \pm 4,6 \mathrm{~A}$ & $+27,3 \pm 3,8$ \\
Coeficiente de Tolerancia al Calor & $5,8 \pm 0,1 \mathrm{~B}$ & $7,8 \pm 0,2 \mathrm{~A}$ & $+2,0 \pm 0,1$ \\
\hline Temperaturas superficial & $\mathrm{CS}$ & $\mathrm{SS}$ & Diferencia SS-CS \\
\hline Lomo & $37,0 \pm 0,2 \mathrm{~b}$ & $+5,6$ \\
Cadera & $36,4 \pm 0,2 \mathrm{~b}$ & $42,6 \pm 0,3 \mathrm{a}$ & $+3,6$ \\
Costado & $36,1 \pm 0,1 \mathrm{~b}$ & $40,0 \pm 0,2 \mathrm{a}$ & $+3,1$ \\
Vena nasal & $35,6 \pm 0,1 \mathrm{~b}$ & $39,2 \pm 0,1 \mathrm{a}$ & $+1,4$ \\
Vena ocular & $35,9 \pm 0,1 \mathrm{~b}$ & $37,0 \pm 0,1 \mathrm{a}$ & $+1,5$ \\
Frente & $36,0 \pm 0,1 \mathrm{~b}$ & $37,1 \pm 0,1 \mathrm{a}$ & $+3,3$ \\
Cráneo & $36,0 \pm 0,1 \mathrm{~b}$ & $39,3 \pm 0,2 \mathrm{a}$ & $+3,0$ \\
Anterior & $35,2 \pm 0,1 \mathrm{~b}$ & $39,0 \pm 0,1 \mathrm{a}$ & $+1,6$ \\
Posterior & $35,2 \pm 0,1 \mathrm{~b}$ & $36,8 \pm 0,1 \mathrm{a}$ & $+1,9$ \\
\hline
\end{tabular}

${ }^{a b}$ Diferente entre columnas indican diferencia significativa $(P \leq 0,01)$

radiación en animales (Okourwa 2015, p. 354), adicionalmente, la exposición directa de las ovejas a la RS, genera una ganancia de energía térmica debido a la absorción de ondas electromagnéticas, explicando la mayor TS observada en ovejas SS (Tabla II).

De acuerdo con los resultados obtenidos al evaluar la TS, existe heterotermia superficial (Tabla II). La diferencia observada al medir la TS en Lomo, Cadera,
Costado, Frente y Base del Cráneo, sugiere un efecto directo de la RS sobre la superficie ubicada hacia la región dorsal; en comparación con la Extremidad Anterior y la Extremidad Posterior. En la región rostral ha sido reportado un mecanismo de enfriamiento selectivo del cerebro en ovinos y cérvidos (Mitchell et al. 1998, p. 279), asociado a una mayor actividad de las venas superficiales para mantener la temperatura del

Tabla III. Vectores medios para describir rangos de variaciones en elementos climáticos y variables fisiológicas mediante el análisis de componentes principales (Vectors mean of the climatic elements and physiological variables by principal component analysis).

\begin{tabular}{|c|c|c|c|c|}
\hline Nivel & \multicolumn{4}{|c|}{ Variable } \\
\hline $\mathrm{TC}\left({ }^{\circ} \mathrm{C}\right)$ & $\operatorname{TGN}\left({ }^{\circ} \mathrm{C}\right)$ & \multicolumn{2}{|c|}{$\mathrm{RS}\left(\mathrm{W} / \mathrm{M}^{2}\right)$} & ITH (Unid.) \\
\hline$\leq 39,0$ & 37,5 & \multicolumn{2}{|c|}{145,7} & 80,0 \\
\hline $39,1-39,5$ & 41,2 & \multicolumn{2}{|c|}{371,4} & 80,4 \\
\hline$\geq 39,6$ & 42,8 & \multicolumn{2}{|c|}{547,6} & 80,7 \\
\hline $\mathrm{TC}\left({ }^{\circ} \mathrm{C}\right)$ & Temperatura lomo $\left({ }^{\circ} \mathrm{C}\right)$ & \multicolumn{2}{|c|}{ Temperatura Cadera $\left({ }^{\circ} \mathrm{C}\right)$} & Temperatura Costado $\left({ }^{\circ} \mathrm{C}\right)$ \\
\hline$\leq 39,0$ & 37,7 & \multicolumn{2}{|c|}{37,2} & 36,7 \\
\hline $39,1-39,5$ & 39,8 & \multicolumn{2}{|c|}{38,2} & 37,7 \\
\hline$\geq 39,6$ & 41,2 & \multicolumn{2}{|c|}{39,0} & 38,2 \\
\hline $\mathrm{FR}(\mathrm{mov} / \mathrm{min})$ & $\operatorname{TA}\left({ }^{\circ} \mathrm{C}\right)$ & $\operatorname{TGN}\left({ }^{\circ} \mathrm{C}\right)$ & $\mathrm{RS}\left(\mathrm{W} / \mathrm{m}^{2}\right)$ & ITH (unidades) \\
\hline $40-60$ & 32,3 & 35,8 & 118,5 & 79,9 \\
\hline $61-80$ & 32,6 & 38,0 & 180,3 & 80,0 \\
\hline $81-120$ & 32,7 & 39,2 & 247,2 & 80,2 \\
\hline $121-150$ & 33,2 & 43,5 & 529,1 & 80,6 \\
\hline$\geq 151$ & 33,5 & 46,6 & 656,0 & 80,9 \\
\hline
\end{tabular}

TGN: Temperatura del globo negro $\left({ }^{\circ} \mathrm{C}\right)$; HR: Humedad relativa (\%); RS: Radiación solar $\left(\mathrm{W} / \mathrm{m}^{2}\right)$; ITH: Índice de temperatura y humedad (unidades); TA: Temperatura ambiente $\left({ }^{\circ} \mathrm{C}\right)$. 
a) Relación entre la temperatura corporal y las variables climáticas

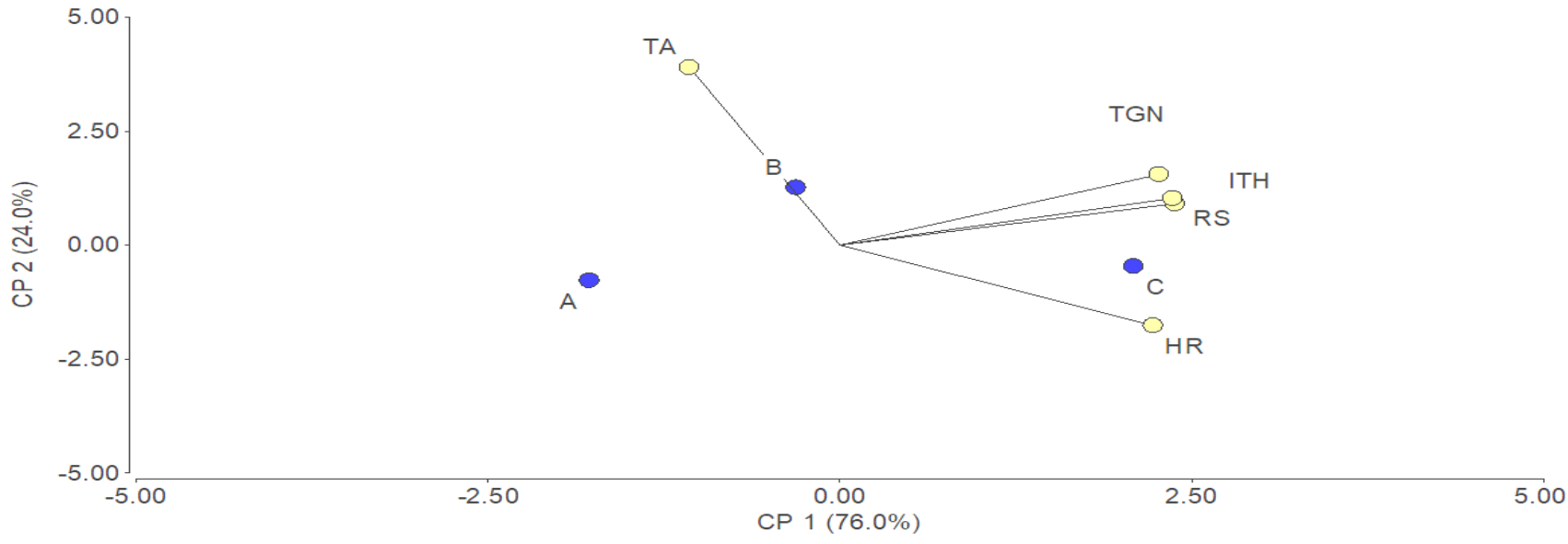

b) Relación entre la temperatura corporal y la temperatura superficial de varias regiones corporales

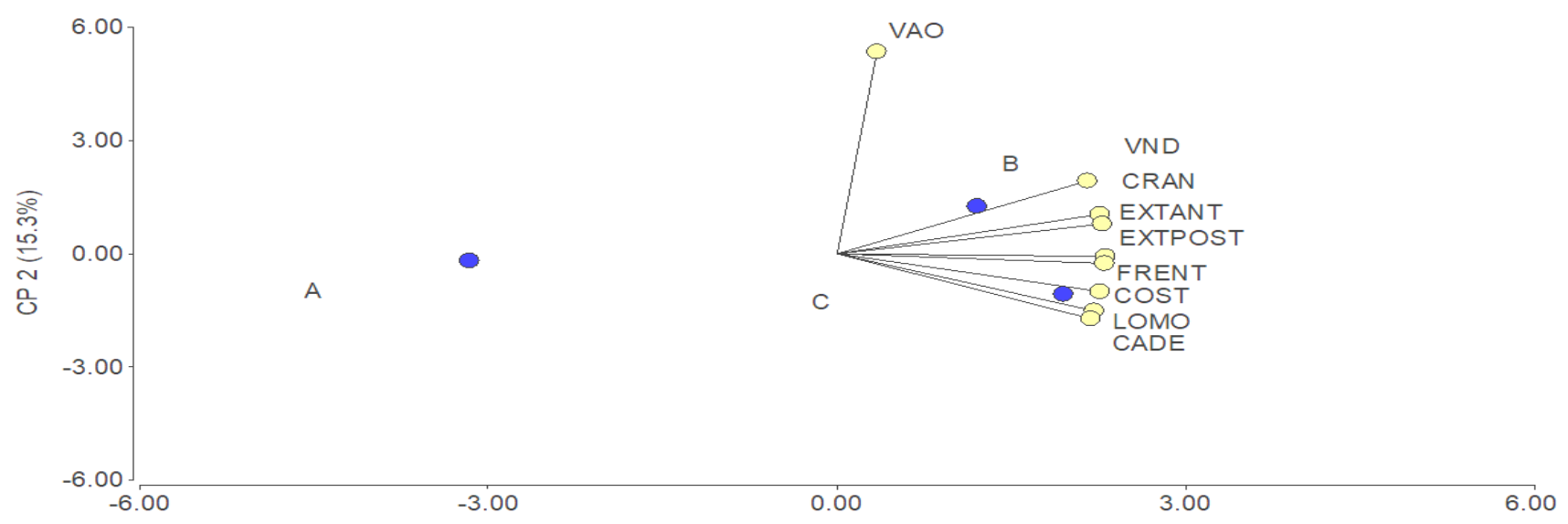

c) Relación entre la frecuencia respiratoria, temperatura corporal y variables climáticas

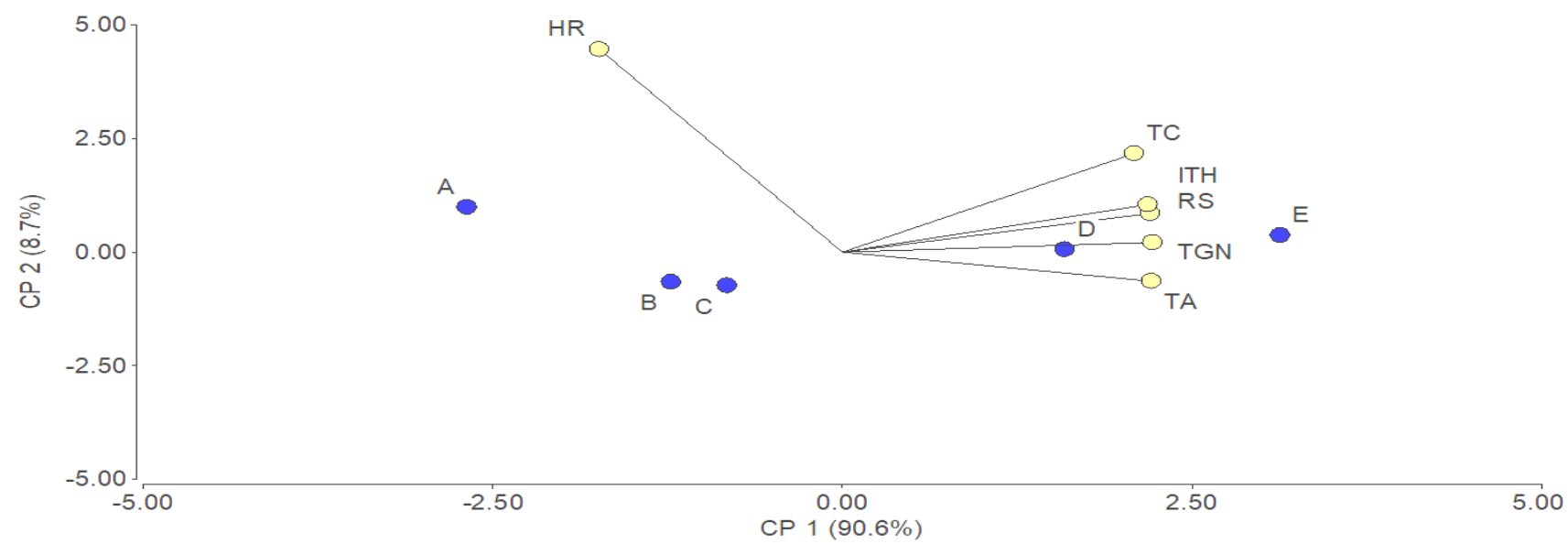

Figura 1. Representación gráfica de la relación entre la temperatura corporal (TC) y las variables climáticas (a), la TC y la temperatura superficial (b) y, la frecuencia respiratoria (FR) y las variables climáticas (c). Rangos de TC: A: TC $\leq 39,0^{\circ} \mathrm{C}$; B: TC entre 39,1-39,5 ${ }^{\circ} \mathrm{C}$; C: TC $\left.\geq 39,6^{\circ} \mathrm{C}\right)$. Rangos de FR: A: FR entre $40-60 \mathrm{mov} / \mathrm{min} ; \mathrm{B}:$ FR entre 61-80 mov/min; C: FR entre 81-120 mov/min; D: FR entre 121-150 mov/min; E: FR >151 mov/min). Temperatura ambiental (TA); Humedad relativa (HR); Radiación solar (RS); Temperatura de globo negro (TGN); Indice de temperatura humedad (ITH); Vena angular ocular (VAO); Vena nasal Dorsal (VND); Base del cráneo (CRAN); EXTANT: Extremidad anterior (EXTANT); Extremidad posterior (EXTPOST); Frente (FRENT); Costado (COST); Lomo (LOMO) y Cadera (CADE) (Graphic representation of the relationship between body temperature (TC) and climatic variables (a), TC and superficial temperature (b) and, respiratory frequency (FR) and climatic variables (c). TC ranges: A: TC $\leq 39.0^{\circ} \mathrm{C} ; \mathrm{B}:$ TC between $39.1-39.5^{\circ} \mathrm{C} ; \mathrm{C}$ : $T C \geq 39.6^{\circ} \mathrm{C}$ ). FR ranges: A: FR between $40-60 \mathrm{mov} / \mathrm{min}$; B: FR between $61-80 \mathrm{mov} / \mathrm{min}$; C: FR between 81-120 mov/min; D: FR between $121-150$ mov/min; E: FR> $151 \mathrm{mov} / \mathrm{min}$ ). Ambient temperature (TA); Relative humidity (RH); Solar radiation (RS); Black globe temperature (TGN); Humidity-temperature index (ITH); Ocular angular vein (OVA); Dorsal nasal vein (VND); Base of the skull (CRAN); EXTANT: Anterior limb (EXTANT); Hind limb (EXTPOST); Front (FRENT); Flank (COST); Loin (LOMO) and Hip (CADE)). 
cerebro por debajo del promedio de la temperatura del cuerpo. Lo anterior podría explicar una menor TS presentada a nivel de la Vena Nasal y la Vena Ocular.

Recientes estudios en vacunos corroboran que la FR, TC e ITH son excelentes predictores de EC (Cerqueira et al. 2016, p. 257). Pese a ello, bajo condiciones tropicales y en animales expuestos al sol, la medición de la TS a nivel del tren posterior del animal pudiera indicar con precisión la TC del animal, lo cual sería de interés para una evaluación rápida de condiciones de EC (Figura 1B). Los vectores medios (Tabla III) indican que cuando la temperatura del Lomo, Cadera y Costado es: $\geq 41,2^{\circ} \mathrm{C} ; \geq 39,0^{\circ} \mathrm{C} \mathrm{y} \geq$ a $38,2^{\circ} \mathrm{C}$, respectivamente, la TC puede ser $\geq 39,6^{\circ} \mathrm{C}$. Bajo estas circunstancias, se podría crear una alerta para promover mecanismos de enfriamiento que garanticen el bienestar térmico de los animales, como el suministro de sombra natural o artificial. Los elementos que mejor explican la variabilidad en la FR son TA, RS, TGN e ITH (CP1: 90,6\%). Valores elevados de FR presentan una relación inversa con la HR (Figura 1C; Tabla III).

\section{CONCLUSIONES}

Las relaciones entre el comportamiento fisiológico y las variables climáticas reflejan la importancia de la RS y la TGN en el bienestar térmico en animales, justificando la necesidad de suministro de sombra. La medición de la TS en Lomo, Cadera y Costado presentó una alta relación con TC. Se confirma la alta capacidad de liberar calor por evaporación respiratoria en la oveja West African y el efecto de la HR en su eficiencia. Los resultados sugieren que el punto de quiebre del ITH en ovinos está $\sim 80$ unidades. Similarmente, valores de temperatura de globo negro $>42,8^{\circ} \mathrm{C}$ y de RS $>529,1$, podrían indicar reducciones del bienestar térmico en ovejas a pastoreo.

\section{AGRADECIMIENTOS}

Los autores agradecemos al proyecto PG-01-83082011/2, financiado por el Consejo de Desarrollo Científico y Humanístico de la Universidad Central de Venezuela (CDCH-UCV).

\section{BIBLIOGRAFIA}

Batista, N L, de Souza, B B, Oliveira, G J C, Roberto, J V B, Ribeiro, T L A \& Silva, R A 2014, 'Tolerância ao calor em ovinos de pelames claro e escuro submetidos ao estresse térmico', Journal of Animal Behaviour Biometeorology, vol 2, no 3, pp. 102-8.

Benezra, MV 1954,'A new index for measuring the adaptability of cattle to tropical conditions', Journal Animal Science, vol 13, Proceeding, p. 1015.

Cerquiera, J O L, Araújo, J P P, Blanco-Penedo, I, Contalapiedra, J, Silvestre, A M D \& Silva, S J C R 2016, 'Predicción de estrés térmico en vacas lecheras mediante indicadores ambientales y fisiológicos', Archivos de Zootecnia, vol 65, no 251, pp. 357-364.

De Sousa Júnior, S C, Morais, D A, de Vasconcelos, A M, Nery, K M, Morais, J H \& Guilhermino, M M 2008,'Características termorreguladoras de caprinos, ovinos e bovinos em diferentes épocas do ano em região semi-árida', Revista Científica de Produção Animal, vol 10, no 2,pp. 127-137.

Di Rienzo, J, Casanoves, F, Balzarini, M, González, L, Tablada, M \& Robledo C 2008, InfoStat, versión 2008, Grupo InfoStat, FCA, Universidad Nacional de Córdoba, Argentina.

Littell, R, Henry, R \& Ammerman, C1998,'Statistical analysis of repeated measures data using SAS procedures', Journal Animal Science, vol 76, no 4, 1216-31.

López, R, Pinto-Santini, L, Perozo, D, Pineda, J, Oliveros, I, Chacón, T., Rossini, M \& Ríos de Álvarez, L2015,'Confort térmico y crecimiento de corderas West African pastoreando con y sin acceso a sombra artificial', Archivos de Zootecnia, vol 64, no 246, pp. 139-46.

Mitchell, J, Thomalla, L \& Mitchell, G 1998,'Histological Studies of the Dorsal Nasal, Angularis Oculi, and Facial Veins of Sheep (Ovisaries)', Journal of Morphology, vol 237, no 3, pp. 275-81.

Núñez-Domínguez, R, Ramírez-Valverde, R, Saavedra-Jiménez LA \& García-Muñiz, J G 2016, 'La adaptabilidad de los recursos zoogenéticos Criollos, base para enfrentar los desafíos de la producción animal', Archivos de Zootecnia, vol 65, no 251, pp. 461-468.

Okourwa, M I 2015,'Effect of coat characteristics on physiological traits and heat tolerance of West African Dwarf sheep in Southern Nigeria', Open Journal Animal Science, vol 5, pp. 351-7.

Quesada, M.; McManus, C. y D'Araújo C.F.A. 2001. 'Tolerância ao calor de duas raças de ovinos deslanados no Distrito Federal', Revista Brasilerira de Zootecnia, vol 30, no 3, pp. 1021-26.

Rocha, R R C, Costa, A P R, Azevedo, D M M R, Nascimiento, H T S, Cardoso, F S, Muratori, M C S \& Lopes, J B 2009, 'Adaptabilidade climática de caprinos Saanen e Azul no Meio-Norte do Brasil', Arquivo Brasileiro de Medicina Veterinária e Zootecnia, vol 61, no 5,pp. $1165-72$

Singh, K M, Singh, S, Ganguly, I, Ganguly, A, Nachiappan, R K, Chopra, A \& Narula, H K 2016,'Evaluation of Indian sheep breeds of arid zone under heat stress condition', Small Ruminant Research, vol 141, pp. 113-7.

Souza, C, Tinoco, I, Baeta, F, Ferreira, W \& Da Silva, R 2002,'Avaliação de materiais alternativos para confecção do termômetro de globo', Ciênciae Agrotecnologia, vol 26, nol, pp. 157-64.

Thom, EC 1959, 'The discomfort index', Weatherwise, vol 12, pp. 57-60. USICLIMA2012,'Unidad de Servicios Integrados Climatológicos para la Investigación en Agricultura y Ambiente', FAGRO-UCV. Cátedra de Climatología Agrícola. 